\title{
Influence of the Main Technological Variables of Cyclical Mechanic-Thermal Processing on the Strain Hardening of Steel Parts
}

\author{
Eleno Alfonso Brindis ${ }^{1}$, Sanjeevkoemar Bissesar ${ }^{1}$, Faizel Abdoel Wahid ${ }^{1}$ and Francisco Tchiquendja Eleno ${ }^{2}$ \\ 1. Department of Mechanical Engineering, Faculty of Science Technology, Anton de Kom University of Suriname, Paramaribo 9212 , \\ Suriname \\ 2. Department of Mechanical Engineering, Faculty of Engineering, University of Matanzas, Matanzas 44740, Cuba
}

\begin{abstract}
The main objective of the present work was to determine the influence of the most important technological variables of CMTP (cyclical mechanic-thermal processing) on the strain hardening in the surface layers of steel parts. For this, it was designed a full factorial plan at two levels of five independent variables that include the whole processing in two and three cycles, the cold-forming degree and force during the plastic deformation (burnishing), and the temperature and time at the given temperature during the aging. Each cycle is composed of plastic deformation at room temperature plus aging. As dependent variables, the degree and penetration depth of strain hardening were evaluated. Based on the appropriately used set of experimental data, it had been fitted an exponential model for each dependent variables and also a two-degree polynomial fitting of in-depth evolution of microhardness profile was obtained. The amount of cycles and the cold-forming degree are the technological variables of CMTP that influence the most on strain hardening, although other variables also are significant. The microhardness profile highlights that during the CMTP, the strain hardening decreases from the outer bound to the transition zone of the surface layers, where it disappears.
\end{abstract}

Key words: Mechanic-thermal processing, strain hardening, surface layers, microhardness.

\section{Introduction}

The CMTP (cyclical mechanic-thermal processing), according Gordienko's definition [1], is a kind of substructural hardening that consists of submitting the parts to several combined cycles of cold plastic deformation and aging in order to obtain a set of high physic-mechanic indicators of quality in their surface layers. This means that, as results of the treatment in the surface layers of parts, a high level of strain hardening with a dislocation cell nanostructure is achieved, which keeps its stability until temperatures are close to the recrystallization temperature of given alloy. At applying the CMTP, an improvement of the mechanical properties and the performance takes place in the metals and their alloys, particularly in those with a BCC (body-centered cubic crystal) structure, as the

Corresponding author: Eleno Alfonso Brindis, Ph.D., professor, research field: materials science and engineering.
AISI 1045 plain mid-carbon steel, used in the present research work.

The strain hardening is the effect of cold plastic deformation process within the surface layers of the metals and alloys, and it is associated with the presence, movement and interaction of dislocations, involving the dislocation density growth. The average distance between dislocations decreases and dislocations start blocking the motion of each other, and these phenomena become the metal harder and stronger.

Panwar et al. [2] also explained the role of the combination of cold work and subsequent aging through analysis of a microalloyed Cu-bearing HSLA (high-strength low alloy) steel; the mechanical properties had been studied at various stages of aging with and without prior cold work at different percent. M'Guil et al. [3] explained the strain hardening through models of observed true stress-true strain curves in the 
work hardening region after tensile tests; they modeled the large plastic deformation behavior and anisotropy evolution in cold rolled BCC steel.

Before testing, the specimens are almost always prepared under different cold-forming degrees by means of:

(a) Rolling [4, 5], these studies report the mechanical property and microstructure of cold rolled low and high carbon steel and its work-hardening behavior during the deformation process;

(b) Burnishing process [6], the obtained results achieved by then show the enhancement in the surface roughness and magnification in surface hardness were achieved, because of application of roller burnishing in mild steel specimens;

(c) High-pressure torsion at room temperature for up to 5 turns, were applied to low-carbon triple-alloyed steel specimens by Marulanda et al. [7]. Microhardness, scanning electron microscopy and $\mathrm{X}$-ray diffraction were used to investigate the hardness and microstructural evolution of the steel. The results show a gradual evolution in both the hardness and the microstructure with increasing numbers of turns.

There are many ways to model strain hardening, each one of them has its own advantages and disadvantages. The strain hardening can be obtained through statistical dislocation density models, dislocation dynamics model and empirical models, as was showed by Prassad [8] in his Master's thesis.

That is precisely why in this work are proposed two new empirical models for establishing the influence of the technological rate of CMTP on strain hardening of steel parts.

\section{Experimental Materials and Procedures}

2.1 The Material under Study and Its Properties in Supply State

According to Standard Specification ASTM A 29/A 29M-04 [9], several grades of plain and low-alloy mid-carbon steels were evaluated, and AISI 1045 steel was chosen, since it is the most economic grade, that allows to fulfill the objective of the present work.

From the employed bar to prepare the specimens, the chemical composition of studied steel was obtained, in \%: $\mathrm{C}=0.47, \mathrm{Mn}=0.70, \mathrm{Si}=0.21, \mathrm{P}=0.04, \mathrm{~S}=0.03$, $\mathrm{Cr}=0.15$ and $\mathrm{Ni}=0.09$.

Moreover, in supply state the following mechanical properties of the tested steel were determined: average hardness, HBS 185 (ASTM E 10-01a) [10]; yield strength, $\sigma_{y}=338 \mathrm{MPa}$; and the tensile (ultimate) strength, $\sigma_{u}=620 \mathrm{MPa}$. The tensile specimens were prepared, following the Standard Test Method for Tension Testing of Metallic Materials [Metric] ASTM E 8M-04 [11].

The specimens for the CMTP application, and later on the microhardness test, were machined to little thick-walled cylinders of $30 \mathrm{~mm}$ outer diameter, 20 $\mathrm{mm}$ inner diameter and $15 \mathrm{~mm}$ wideness.

\subsection{Machines, Devices, Tools and Instruments}

The macrohardness measuring of supply state material was done using the HB-3000B Manual Digital Display Brinell Hardness Tester/Metal Brinell Durometer.

The surface roughness was evaluated using the comparison method by means of the ZUZI model 173/2.

Mounted specimens for the microhardness measurement were ground in STRUERS Labopol-21 Machine, and lastly they were polished in the STRUERS Labopol-5 Machine.

The microhardness measurement had been developed in the metallographic microscope model SCHENK TREBEL, with a Vickers diamond pyramid indenter and its own devices for fixing the specimens.

A cutting disk for the STRUERS Labopol-5 Machine was used.

Both single point-brazed cutting tools P10 and P20 (ISO R513-1975E) [12] for the preliminary specimens sizing had been used.

For the drilling operation ware employed a 60 degree center drilling bit, M2 drill rods $(10$ and $18 \mathrm{~mm}$ 
diameter); and a M2 reamer (20 $\mathrm{H} 8$ diameter).

The burnishing operation was made using a Yamasa Sx8-1.2 single roll type roller burnishing tool.

A set of carborundum (SiC) emery paper $(240,320$ and 600, according to ASTM E 3-01 [13]) was employed for the specimens grinding, while for the polishing operations one used both diamond paste in sizes $6 \mu \mathrm{m}$ and $1 \mu \mathrm{m}$.

\subsection{Design and Run of Experiments}

CMTP experiments were carried out on external surfaces of little thick-walled cylinders. In the experiments, the work pieces were burnished after turning on lathe, in the same set-up. While burnishing, the roller burnishing tool is fixed in its own coupled-dynamometer. That dynamometer is capable of measuring three force components. The $\mathrm{z}$ component was taken as the burnishing force.

After each stage of burnishing, the specimens have been subjected to the aging for completing each cycle, that is, it is in order to organize the dislocation, forming sub-grains and sub-borders in all grains by means of cell fragmentation (Gordienko [1]).

Independently of the applied variables' combination, the surface roughness were improved till $R_{a}=0.32 \ldots$ 0.16 micrometers after burnishing process and remains at the same level after aging stage. It was not formed any oxide husk over the surface layer, inasmuch as the temperature never rose higher than $200^{\circ} \mathrm{C}$.

In order to reveal a clear picture of CMTP, a series of experiments were conducted on prepared specimens from selected AISI 1045 plain carbon steel. A full factorial orthogonal array had been chosen, for five factors at two-level, that required $32\left(2^{5}\right)$ experiments (Table 1).

\section{Results and Discussion}

During the cold processing of ductile alloys (as AISI 1045 steel in supply state), the strain hardening behavior, the degree and depth of strain hardening are reported mainly as result of plastic deformation, using
Table 1 Factors and levels chosen for experimentation (common for both degree and depth of strain hardening experiments).

\begin{tabular}{lll}
\hline Factors & $\begin{array}{l}\text { Low level } \\
(-1)\end{array}$ & High level (1) \\
\hline Amount of cycles $(A)$ & 2 & 3 \\
Cold-forming degree $(\lambda), \%$ & 4 & 7 \\
Force $(F), \mathrm{kN}$ & 0.8 & 1.5 \\
Temperature $(t),{ }^{\circ} \mathrm{C}$ & 100 & 200 \\
Time $(\tau), \mathrm{h}$ & 1.0 & 1.5 \\
\hline
\end{tabular}

different models and theories for its foundation, thus González et al. [14] established a connection between the coefficient of strain hardening, mechanical strength and admissible thickness tolerances in steel sheets, that it is an important contribution for materials testing.

The empirical models are quite a bit used, and they describe the flow stress in terms of power laws as a function of strain with many empirical constants. One of the simplest approaches is the Hollomon equation which describes the flow stress as a simple power law in strain, it is applied in several recent works [15]; even in these works the true stress-strain curve and its polynomial fit curve of a cold rolled low-carbon steel had been compared. The Hollomon equation is given as

$$
\sigma=K \varepsilon^{n_{H}}
$$

where, $\sigma$ is the true stress, $K$ is the coefficient of deformation strengthening, $\varepsilon$ is the true strain and $n_{H}$ is the index of strain hardening.

The results of above mentioned research works and other with more complicated models depend of tensile test and they do not show any explicit physic-mechanic indicator of quality in the surface layers of tested material.

In the present work, an empirical model had been used, that evaluates the strain hardening, as the main physical-mechanical quality indicator in the surface layers of the AISI 1045 plain mid-carbon steel. Here both degree and penetration depth dependencies of the strain hardening on the main cold plastic deformation and aging variables were analyzed, after specimen were submitted to CMTP.

Table 2 shows the experimental matrix and results, for both features of strain hardening: degree $(N)$, and depth $(h)$. 
Table 2 Experimental matrix and results for degree and penetration depth of strain hardening.

\begin{tabular}{|c|c|c|c|c|c|c|c|}
\hline \multirow{2}{*}{$\begin{array}{l}\mathrm{N}^{\mathrm{o}} \\
\text { exp. }\end{array}$} & \multicolumn{5}{|c|}{ Factors } & \multirow{2}{*}{$\begin{array}{l}\text { Experimental } \\
N(\%)\end{array}$} & \multirow{2}{*}{$\frac{\text { Results }}{h(\mathrm{~mm})}$} \\
\hline & $A$ & $\lambda(\%)$ & $F(\mathrm{kN})$ & $t\left({ }^{\circ} \mathrm{C}\right)$ & $\tau(\mathrm{h})$ & & \\
\hline 1 & 3 & 7 & 1.5 & 200 & 1.5 & 48.000 & 0.850 \\
\hline 2 & 2 & 7 & 1.5 & 200 & 1.5 & 70.000 & 0.500 \\
\hline 3 & 3 & 4 & 1.5 & 200 & 1.5 & 34.000 & 0.750 \\
\hline 4 & 2 & 4 & 1.5 & 200 & 1.5 & 53.000 & 0.450 \\
\hline 5 & 3 & 7 & 0.8 & 200 & 1.5 & 44.000 & 0.800 \\
\hline 6 & 2 & 7 & 0.8 & 200 & 1.5 & 65.000 & 0.450 \\
\hline 7 & 3 & 4 & 0.8 & 200 & 1.5 & 33.000 & 0.700 \\
\hline 8 & 2 & 4 & 0.8 & 200 & 1.5 & 52.000 & 0.400 \\
\hline 9 & 3 & 7 & 1.5 & 100 & 1.5 & 55.000 & 0.800 \\
\hline 10 & 2 & 7 & 1.5 & 100 & 1.5 & 72.000 & 0.500 \\
\hline 11 & 3 & 4 & 1.5 & 100 & 1.5 & 39.000 & 0.650 \\
\hline 12 & 2 & 4 & 1.5 & 100 & 1.5 & 58.500 & 0.400 \\
\hline 13 & 3 & 7 & 0.8 & 100 & 1.5 & 46.000 & 0.750 \\
\hline 14 & 2 & 7 & 0.8 & 100 & 1.5 & 67.000 & 0.400 \\
\hline 15 & 3 & 4 & 0.8 & 100 & 1.5 & 36.000 & 0.600 \\
\hline 16 & 2 & 4 & 0.8 & 100 & 1.5 & 58.000 & 0.350 \\
\hline 17 & 3 & 7 & 1.5 & 200 & 1.0 & 52.000 & 0.850 \\
\hline 18 & 2 & 7 & 1.5 & 200 & 1.0 & 75.000 & 0.500 \\
\hline 19 & 3 & 4 & 1.5 & 200 & 1.0 & 36.500 & 0.650 \\
\hline 20 & 2 & 4 & 1.5 & 200 & 1.0 & 55.000 & 0.400 \\
\hline 21 & 3 & 7 & 0.8 & 200 & 1.0 & 47.000 & 0.700 \\
\hline 22 & 2 & 7 & 0.8 & 200 & 1.0 & 68.000 & 0.400 \\
\hline 23 & 3 & 4 & 0.8 & 200 & 1.0 & 33.000 & 0.600 \\
\hline 24 & 2 & 4 & 0.8 & 200 & 1.0 & 56.000 & 0.350 \\
\hline 25 & 3 & 7 & 1.5 & 100 & 1.0 & 53.500 & 0.750 \\
\hline 26 & 2 & 7 & 1.5 & 100 & 1.0 & 78.000 & 0.450 \\
\hline 27 & 3 & 4 & 1.5 & 100 & 1.0 & 44.500 & 0.600 \\
\hline 28 & 2 & 4 & 1.5 & 100 & 1.0 & 59.000 & 0.400 \\
\hline 29 & 3 & 7 & 0.8 & 100 & 1.0 & 52.000 & 0.700 \\
\hline 30 & 2 & 7 & 0.8 & 100 & 1.0 & 75.000 & 0.450 \\
\hline 31 & 3 & 4 & 0.8 & 100 & 1.0 & 40.000 & 0.550 \\
\hline 32 & 2 & 4 & 0.8 & 100 & 1.0 & 52.500 & 0.300 \\
\hline
\end{tabular}

The values of " $N$ " and " $h$ " were obtained after measuring the microhardness across the surface layers of specimens during each experiment and then using the following classic dependencies:

$$
\begin{gathered}
N=\left[\left(H_{\mu \max }-H_{\mu o}\right) / H_{\mu o}\right]^{*} 100 \% \\
\text { DepthSH }=h=f\left(H_{\mu \max }, H_{\mu o}\right)
\end{gathered}
$$

where, $H_{\mu \max }$ characterizes the achieved level of strain hardening that is the maximum value of microhardness in the outer edge of surface layer at its deformation zone, $H_{\mu o}$ corresponds to the microhardness value at the transition zone of surface layer, and it is taken when the value of measured microhardness remains almost constant, independently from the increasing distance of indentation towards the specimen core (Fig. 1).

As results of this study two empirical relationships among the input- main technological variables of the processing rate and output- two of the major physic-mechanic features of the strain hardening were fitted.

Once established the final fitted models, the influences of input (independent variables) on output (dependent variables) were analyzed. 


\subsection{Degree of Strain Hardening}

$$
N=99.335 A^{-0.950} \lambda^{0.488} F^{0.112} t^{-0.120} \tau^{-0.135}
$$

The joint assessment of Tables 2 and 3, as well as Eq. (4), one can explain that:

The greatest influence on the magnitude " $N$ " is exhibited by the amount of cycles $(A)$. In Table 2 , experimentally it is showed that at the low level (2 cycles) correspond the highest values of " $N$ ", this situation is consistent with the statistics depicted in Table 3, where the estimated coefficient for variable " $A$ " has a negative value, as well as in the Student distribution, whose absolute value is the highest (this means the most significant variable). In this sense, another significant detail is that its $p$ Value is the lowest.

From the metallographycal point of view, this phenomenon is because of the difference between the added by the cycle maximal microhardness " $H_{\mu c}$ " and the microhardness in the transition zone " $H_{\mu}$ " of the surface layers, consecutively decreases after each cycle due to the strengthening of material by dislocation mechanism.

The influence in the magnitude " $N$ " of the cold-forming degree $(\lambda)$ and the force $(F)$, one can explain as: the greater " $\lambda$ " and " $F$ " values, the greater the magnitude " $N$ ". An increase of the cold-forming degree and proportionally of the force during the plastic deformation process (burnishing), involves major amount of dislocation in the surface layers. According to the results of Xue et al. [16], at the beginning of deformation, plastic deformation caused by applied force prompts out pouring dislocation movement along gliding plane and lead to the dislocation multiplication. It further makes the dislocation density bigger and bigger, the main and second slip system of the parallel dislocation interaction blocks the dislocation further moving, and so it produces remarkable strain hardening.

Gordienko [1] showed that after aging, the blocked into grains dislocations and other defects clump together to form thousands sub-grains in each grain. These sub-grains achieve a cellular dislocation structure that raises the performance of machine parts during the CMTP.

The aging temperature " $t$ " strongly influences on the magnitude " $N$ ", while greater is the value of temperature, lesser is the magnitude " $N$ ", as it is showed in Table 2. At the same time, this statement can be corroborated in Table 3, where the estimated coefficient for variable " $t$ " has sign "“" and correspondingly the value of tStat exhibits the same sign; moreover, the pValue of variable " $t$ " is the third lowest of the whole model.

The aging time " $~ \tau$ " is the variable that less influences in the magnitude " $N$ ". Although in lesser degree, the aging time experimentally and statistically follows the behavior of aging temperature, with regard to dependent variable " $N$ ".

The greater values of aging temperature and time benefit the recovery process inside the surface layers which, in turn, mean that the degree of strain hardening decreases by lowering the levels of microhardness in the surface layers.

\subsection{Penetration Depth of Strain Hardening}

$$
h=0.053 A^{1.291} \lambda^{0.339} F^{0.192} t^{0.110} \tau^{0.191}
$$

At analyzing the outcomes from Tables 2 and 4, as well as Eq. (5), it is possible to realize that the greater values of every independent variables generate the greater values of dependent variable " $h$ ". Here, as in the case mentioned above, the amount of cycles (here with sign "+") and the cold-deformation degree have

Table 3 The fitting results of degree of strain hardening.

\begin{tabular}{lllll}
\hline Variable & $\begin{array}{l}\text { Estimate } \\
\text { Coef. }\end{array}$ & SE & tStat & pValue \\
\hline Intercept & 3.82780 & 0.22116 & 17.308 & $8.6602 \mathrm{e}-16$ \\
$A$ & -0.9504 & 0.03965 & -23.97 & $2.9705 \mathrm{e}-19$ \\
$\lambda$ & 0.48794 & 0.028728 & 16.985 & $1.3603 \mathrm{e}-15$ \\
$F$ & 0.11157 & 0.025575 & 4.3625 & 0.00018073 \\
$t$ & -0.12030 & 0.023194 & -5.1866 & $2.0548 \mathrm{e}-05$ \\
$\tau$ & -0.13506 & 0.039650 & -3.4062 & 0.0021509 \\
\hline
\end{tabular}

Number of observations: 32, Error degrees of freedom: 26. Root mean squared error: 0.0455 .

R-squared: 0.973, Adjusted R-Squared 0.967.

F-statistic vs. constant model: $184, \mathrm{p}$-value $=1.97 \mathrm{e}-19$. 
Table 4 The fitting results of penetration depth of strain hardening.

\begin{tabular}{lllll}
\hline Variable & $\begin{array}{l}\text { Estimate } \\
\text { coef. }\end{array}$ & SE & tStat & pValue \\
\hline Intercept & -2.94330 & 0.146320 & -20.116 & $2.2596 \mathrm{e}-17$ \\
$A$ & 1.29150 & 0.044656 & 28.921 & $2.6709 \mathrm{e}-21$ \\
$\lambda$ & 0.33907 & 0.032355 & 10.479 & $7.9243 \mathrm{e}-11$ \\
$F$ & 0.19175 & 0.028804 & 6.6571 & $4.6208 \mathrm{e}-07$ \\
$t$ & 0.11050 & 0.026122 & 4.2300 & 0.00025604 \\
$\tau$ & 0.19148 & 0.044656 & 4.2878 & 0.00021998 \\
\hline
\end{tabular}

Number of observations: 32, Error degrees of freedom: 26. Root mean squared error: 0.0512 .

R-squared: 0.975, Adjusted R-Squared 0.971.

F-statistic vs. constant model: 205, p-value $=4.96 \mathrm{e}-20$.

the greatest influence on the dependent variable " $h$ "; but in this case, it is different the ranking for the other variables, since in descending hierarchical order go on the force, the time and the temperature. Note that between the time and temperature just a slight difference of values.

In the case of penetration depth of strain hardening, at increasing the amount of cycles also are increased the progressive effect of cold-forming degree and of applied force after each cycle [17]; this leads to greater enlargement of the deformation zone of the surface layers towards the specimen core.

The greater values of aging time and temperature, in spite of benefiting the recorvery process, moreover, they enhance the diffusion and self-diffusion processes inside the surface layers, therfore, they also contribute to increase the penetration depth of strain hardening.

\subsection{In-depth Evolution of Microhardness Profile}

It is usual, in many research works within the fields of materials science and engineering, to establish several dependencies that involve the microhardness as the starting point for the studies of strain hardening through its level, degree and penetration depth. These studies cover the most varied materials and treatments applied during the manufacturing processes of machine parts: Alfonso [18], for quenched and tempered steel; Nobre et al [19] proposed methodology for estimating the local yield stress in work-hardened surface layers; Marulanda et al. showed that full homogeneity of the microstructure across the tested steel disks, and therefore of the microhardness requires to apply pressures higher than $6.0 \mathrm{GPa}$. In-depth evolution of microhardness profile is presented in those works.

Fig. 1 depicts the in-depth evolution of microhardness profile along the processed surface layers, from their outer bound of deformation zone to the transition zone, with prediction bounds (Pred bnds) of $95 \%$. The value of microhardness, decreases continuously from the outer surface, where its maximum value " $H_{\mu \max }$ " is found, to the interior of material to its basic value " $H_{\mu o}$ ", further this value remains practically constant, which means that transition zone is not affected by the strain hardening.

In Fig. 1, the microhardness is given in Megapascal (MPa), and the depth of strain hardening (DepthSH) is given in millimeters $(\mathrm{mm})$.

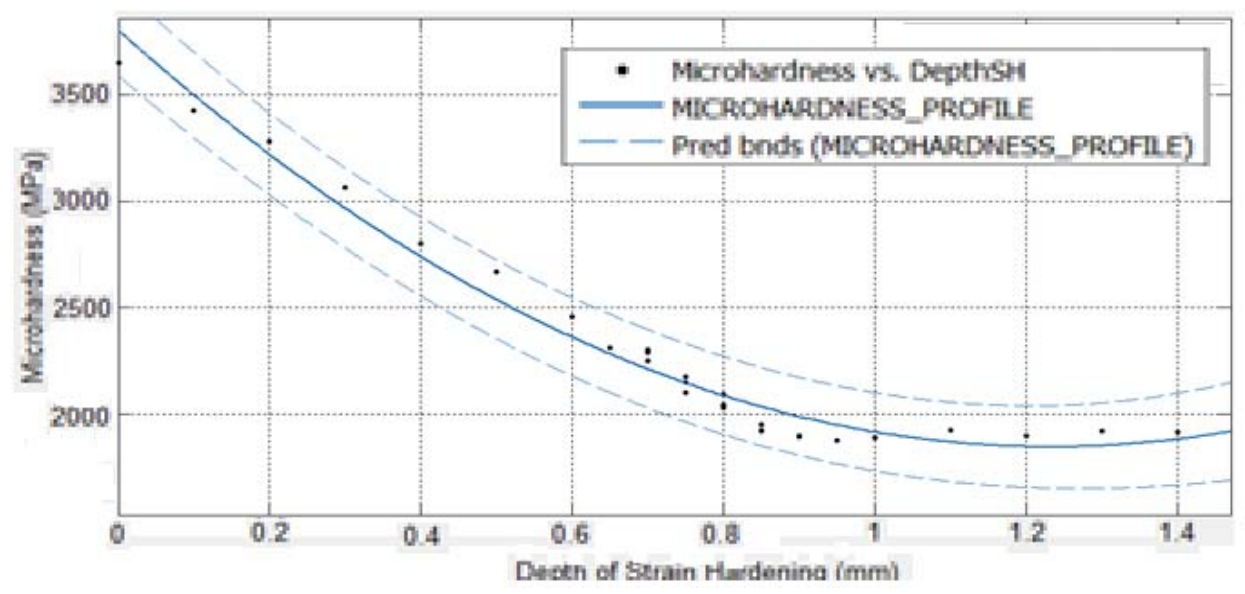

Fig. 1 Microhardness profile of AISI 1045 plain carbon steel after three cycles of mechanic-thermal processing. 


\section{Conclusions}

Once finished this research, it was possible to reach the following conclusions:

(1) The CMTP increases, strengthens and stabilizes the strain hardening in the surface layers of steel parts at raising their main physic-mechanic indicators of quality.

(2) Both obtained models for degree and penetration depth of strain hardening allow to predict these two physic-mechanic quality indicator of the surface layers through the applied technologycal rate of CMTP.

(3) There are many empirical models for steel machining, cold and heat forming, but the CMTP of steel are not sufficiently investigated through empirical models.

(4) Empirical models can be used in order to have a sense of how CMTP parameters influence on the main characteristics of the strain hardening, saving time and resources during the research works.

(5) The microhardness profile highlights that during the CMTP the strain hardening decreases from the outer bound to the transition zone of the surface layers, where it disappears.

\section{Acknowledgment}

This work was supported by the Department of Mechanical Engineering of Anton de Kom University of Suriname.

\section{References}

[1] Gordienko, L. K. 1973. "Substructural Hardening of Metals and Alloys." Nauka Issue, Moscow: 224. (in Russian)

[2] Panwar, S., Goel, D. B., and Pandey, O. P. 2007. "Effect of Cold Work and Aging on Mechanical Properties of Copper Bearing Microalloyed HSLA-100 (GPT) Steel." Bulletin of Material Science 30 (2): 73-9.

[3] M'Guil, S., Wen, W., Ahzi, S., and Gracio, J. J. 2011. "Modeling of Large Plastic Deformation Behavior and Anisotropy Evolution in Cold Rolled BCC Steels Using the Viscoplastic $\phi$-Model-Based Grain-Interaction." Mater. Sci. Eng. A 528 (18): 5840-53.
[4] Zemčík, O., Chladil, J., Otoupalík, J., and Sedlák, J. 2013. "Changes in the Surface Layer of Rolled Bearing Steel." Journal Proceedings in Manufacturing Systems 8 (2): 99-104.

[5] Wang, S. F., Peng, Y., and Li, Z. J. 2013. "Work Hardening and Deformation Mechanism of Cold Rolled Low Carbon Steel." Research Journal of Applied Sciences, Engineering and Technology 5 (3): 823-8.

[6] Malleswara, R. J. N., Chenna K. R. A., and Rama R. P. V. 2011. "The Effect of Roller Burnishing on Surface Hardness and Surface Roughness on Mild Steel Specimens." International Journal of Applied Engineering Research 1 (4): 777-85.

[7] Marulanda, D. M., Wongsa-Ngam, J., and Langdon T. G. 2014. "Microstructural Evolution and Microhardness in Low Carbon Steel Processed by High-Pressure Torsion." Journal of Materials Research and Technology 3 (4): 344-8.

[8] Prassad, G. V. S. S. 2007. "An Improved Dislocation Density Based Work Hardening Model for Al-alloys." M.Sc. Thesis, Rheinisch-Westfälischen Technischen Hochschule.

[9] ASTM A 29/A 29M-04. 2004. "Standard Specification for Steel Bars, Carbon and Alloy, Hot-Wrought, General Requirements."

[10] ASTM E 10-01a. 2001. "Standard Test Method for Brinell Hardness of Metallic Materials."

[11] ASTM E 8M-04. 2004. "Standard Test Method for Tension Testing of Metallic Materials [Metric]."

[12] ISO R513-[1975 (E)] System "Application of Carbides for Machining by Chip Removal."

[13] ASTM E 3-01. 2001. "Standard Guide for Preparation of Metallographic Specimens."

[14] González, R., García, J. O., Barbés, M. A., Quintana, M. J., Verdeja, L. F., and Verdeja, J. I. 2010. "Ultrafine Grained HSLA Steels for Cold Forming." Journal of Iron and Steel Research International 17 (10): 50-6.

[15] Chen, L., and Deng, M. 2011. "Study on Algorithm of Statistics for Bolts Information of Steel Bridge and Iron Tower Based on Assembly Feature." Advances in Information Sciences and Service Sciences 3 (10): 1-11.

[16] Xue, Z. Y., Zhou, S., and Wei, X. C. 2010. "Influence of Pre-transformed Marten Site on Work-Hardening Behavior of SUS 304 Metastable Austenitic Stainless Steel.” J. Iron Steel Res. Int. 17 (3): 51-5.

[17] Delgado, S. Y. 2014. "Sub-microcrystalline Structural State Formation of Low Carbon Steel and Steel 
Influence of the Main Technological Variables of Cyclical Mechanic-Thermal Processing on the Strain Hardening of Steel Parts

Composites, during Their Thermomechanical Processing." Ph.D. Thesis, Ural Institute of Metal Physics. (in Russian)

[18] Brindis, E. A. 1990. "Microstructure and Properties Formation in the Surface Layers of Quenched and Tempered Steel, after Its Finish Turning.” Ph.D. Thesis,
Moscow Institute of Chemical Machinery-Construction. (in Russian)

[19] Nobre, J. P., Dias, A. M., and Kornmeier, M. 2004. "An Empirical Methodology to Estimate a Local Yield Stress in Work-hardened Surface Layers." Society for Experimental Mechanics 44 (1): 76-84. 